\title{
Positive white nipple sign on esophageal varices: Not a just bystander; our experience
}

\section{다)(앙}

\author{
Authors \\ Patel $^{2}$, Nisharg Patel ${ }^{1}$ \\ Institutions \\ 1 SIDS Hospital \& Research Centre - Department of \\ Gastroenterology \& Hepatology, Surat, India \\ 2 SIDS Hospital \& Research Centre - GI Endoscopy, Surat, \\ India
}

Ritesh Prajhapati ${ }^{1}$, Mohit Sethia ${ }^{2}$, Pankaj Desai ${ }^{2}$, Mayank Kabrawala ${ }^{1}$, Rajiv Mehta ${ }^{1}$, Subhash Nandwani ${ }^{1}$, Chintan

submitted 7.5.2021

accepted after revision 28.7.2021

Bibliography

Endosc Int Open 2021; 09: E1837-E1840

DOI $10.1055 / a-1578-2223$

ISSN 2364-3722

(c) 2021. The Author(s).

This is an open access article published by Thieme under the terms of the Creative Commons Attribution-NonDerivative-NonCommercial License, permitting copying and reproduction so long as the original work is given appropriate credit. Contents may not be used for commercial purposes, or adapted, remixed, transformed or built upon. (https://creativecommons.org/licenses/by-nc-nd/4.0/)

Georg Thieme Verlag KG, Rüdigerstraße 14,

70469 Stuttgart, Germany

Corresponding author

Pankaj Desai, Surat Institute of Digestive Sciences - EUS and Endoscopy, Majura Gate Road, Surat Surat Gujarat 395002, India

Fax: +919824100681

drp_desai@hotmail.com

\section{ABSTRACT}

Background and study aims The goal of this study was to assess whether a white nipple sign on esophageal varices is of no prognostic significance or mandates more attention. Patients and methods We retrospectively analyzed data from 2601 patients undergoing upper gastrointestinal endoscopy for variceal bleed from January 2008 to January 2020. Intraprocedural events like onset of active spurt while performing endoscopy, active spurt while attempting to band the varix with a nipple, need for rescue glue therapy required to control bleed in cases of failed endoscopic variceal ligation (EVL), slipping of band and rebleed despite successful band application, need for emergency intubation, and pulmonary aspiration-related complications were noted.

Results A total of 2601 patients underwent endoscopy for variceal bleeding. Of them, 631 had a positive white nipple sign. Of that subgroup, 137 (21.7\%) patients developed active spurt during endoscopy. In patients with the white nipple sign, $12.3 \%$ required endotracheal intubation and $6.7 \%$ developed aspiration pneumonia, which were significantly higher than in those without the sign. Rescue glue injection in esophageal varices was needed in $5.6 \%$ as compared to $0.6 \%$ in those without white nipple.

Conclusions The white nipple sign is not only a predictor of recent bleed, but it carries statistically significant increased risk of intraoperative bleeding, need for endotracheal intubation, esophageal glue injections, and aspiration-related complications. Therefore, it is not just a bystander, but rather, a sign of increased danger and a need to be more vigilant with patient management.

\section{Introduction}

Esophageal varices form as a consequence of portal hypertension preferentially in the submucosa of the lower esophagus. Rupture and bleeding from esophageal varices are major complications of portal hypertension and are associated with a high mortality rate.

The most commonly seen stigmata are red-colored signs, including red wale marks (longitudinal red streaks on varices that resemble red corduroy wales), cherry red spots, and hematocystic spots (raised discrete red spots on the surface of varices that appear as blood blisters) [1].

A less commonly recognized stigma of recent variceal hemorrhage is a white nipple-like projection from a varix into the esophageal lumen. This is a platelet-fibrin plug. Some call it a thrombus. It is referred to as the "white nipple sign: and is a stigma of a site of recent bleeding [2]. Recognition of this stig- 
ma of recent bleeding is necessary to manage the varix endoscopically to prevent further variceal bleeding or intraprocedural bleeding and its incipient complications [3]. This sign is not well described in the literature and is believed that it is only a stigma of recent hemorrhage.

In our practice, however, we have found several instances of intraprocedural bleed in our earliest cases when we saw the nipple. In our experience, a white nipple sign is not only a stigma of recent hemorrhage but also causes torrential intraprocedural bleed with increased risk of aspiration. Hence, we retrospectively analyzed our data and compared intraprocedural events between patients with and without a white nipple sign.

The aim of this study was to assess whether a white nipple sign on esophageal varices is of no prognostic significance or mandates more attention.

\section{Patients and methods}

A total of 2601 patients underwent upper gastrointestinal endoscopy for variceal bleeding from January 2008 to January 2020. We retrospectively evaluated these data. Medical management was done as per established guidelines. The patients underwent endoscopy within 12 hours of admission. From 2008 all endoscopy reports were made with a predefined proforma. Red color signs and signs of recent hemorrhage were specifically mentioned in all endoscopy reports about variceal bleed.

During endoscopy, we injected glue in all gastric varices (GOV 1, GOV 2) and isolated gastric varices (IGV) [4]. The dose of glue was $0.5 \mathrm{~mL}$ per aliquot followed by 1.5 to $3 \mathrm{~mL}$ of distilled water. The esophageal varices were then banded using a multiband ligation device. We thoroughly looked for any white nipple sign on any varix. If a nipple was found, we carefully tried to evade it during scope movement. If the varix with the nipple crossed the gastroesophageal junction, we first injected cyanoacrylate glue in the GOVs. After blocking the gastric varices, including the one with the nipple sign, we subjected the patient to EVL. During EVL we applied the first band on the varix with the nipple. If the nipple was located higher up beyond the mid-esophagus, we applied two to three bands distally and then applied the fourth or fifth band on the varix with nipple. We made sure that we had at least two bands remaining on the barrel before applying a band on the nipple. The rationale was that if the band slipped from the nipple, we had additional bands left to immediately apply again on the same varix. While targeting the nipple, the band was placed on the nipple such that the nipple was located in the distal half of the semicircle of the barrel and then suction was applied. The suction usually pulls the nipple upward and to the center and the band is released in one go after a complete red out. Suction once applied was not released, because that could cause massive bleeding. If the band was not applied as desired or if active spurting started before banding during the procedure, we reattempted banding on the spurter after giving tamponade with the cap on the nipple site. If banding failed, we intubated the patient and reattempted EVL.
After June 2019, all patients who were positive for a white nipple sign were electively intubated before proceeding to endotherapy. If EVL failed, we would remove the banding unit and inject glue. The first injection would be done in the varix just below the nipple and if bleeding was not controlled, then injection directly into the nipple was done. If all measures failed, Denis Ella Stent placement was done.

Intraprocedural events such as onset of active spurt while performing endoscopy, active spurt while catching nipple into the band, need for rescue glue therapy required to control bleed in case of failed EVL, slipping of band and rebleeding despite successful band application, need for emergency intubation, and aspiration-related complications were noted.

\section{Statistical analysis}

Categorical data were presented as frequency and percentage. Comparison of categorical variables were compared using chisquared test. $P<0.05$ was considered statistically significant. All statistical analysis was performed using SPSS v 16.0 software (SPSS Inc., Chicago, Illinois, United States) ( Fig.1, > Fig. 2, - Fig. 3).

\section{Results}

A total of 2601 patients with variceal bleeding were the study population. The etiology of portal hypertension included cirrhosis in 2210, non-cirrhotic portal fibrosis (NCPF) in 119, and extrahepatic portal venous obstruction (EHPVO) in 272.

A positive white nipple sign was noted on esophageal varices in 631 patienbts $(24.3 \%$ ) ( Table 1 ). A positive white nipple sign was not found twice in any of the patients. An active spurt started from the nipple during the procedure in 137 patients (21.7\%). Varices with a white nipple sign were managed successfully with EVL in 594 of 631 patients (94.1\%). In 542 events $(85.9 \%)$, a nipple was found in the mid-esophagus, and hence, the band was first applied on the same varix but distal to the nipple, followed by band application on the nipple. In 89 patients (14.1\%), band slippage occurred despite successful band application. Repeat band application was successful in 52 of 89 patients (58.4\%). In 37 of 89 events (41.6\%), repeat band application was not successful. Of these 37 events, 35 had poor visibility because of active spurt. Cyanoacrylate glue was used in patients in whom EVL failed to secure hemostasis. Of the 37 failed events, hemostasis could be achieved in 35 patients with glue. Two patients required placement of a Denis Ella stent to achieve hemostasis. The mean number of glue attempts required to achieve hemostasis in the nipple group was 3.6 (range, 1$9)$. In the non-nipple group, only 12 of $1970(0.6 \%)$ required use of glue to achieve hemostasis in bleeding esophageal varices.

Intraprocedural endotracheal intubation was needed in 78 of 631 patients $(12.3 \%)$ in the nipple-positive group compared with 112 of 1970 (5.2\%) in the non-nipple group $(P<0.0001)$. In the nipple group 19 of 78 intubations were elective. Aspiration pneumonitis developed in 42 of 631 events (6.7\%) in the nipple group compared with 59 of 1970 events $(0.3 \%)$ in the non-nipple group $(P<0.0001)$. Of the 42 aspiration events, 39 occurred 


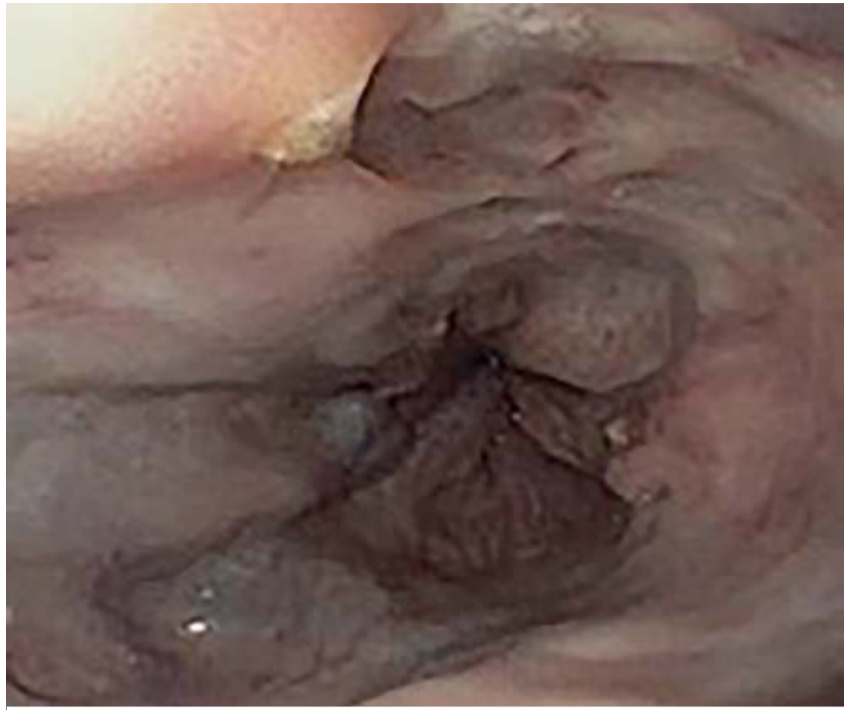

Fig. 1 Varices showing white nipple sign at different locations.

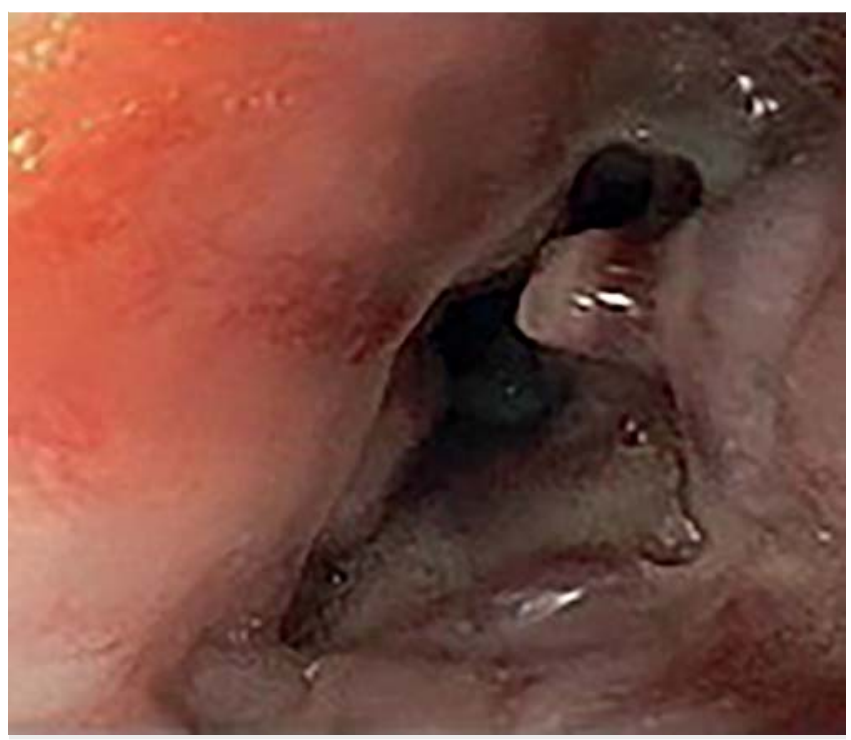

- Fig. 2 Varices showing white nipple sign at different locations.

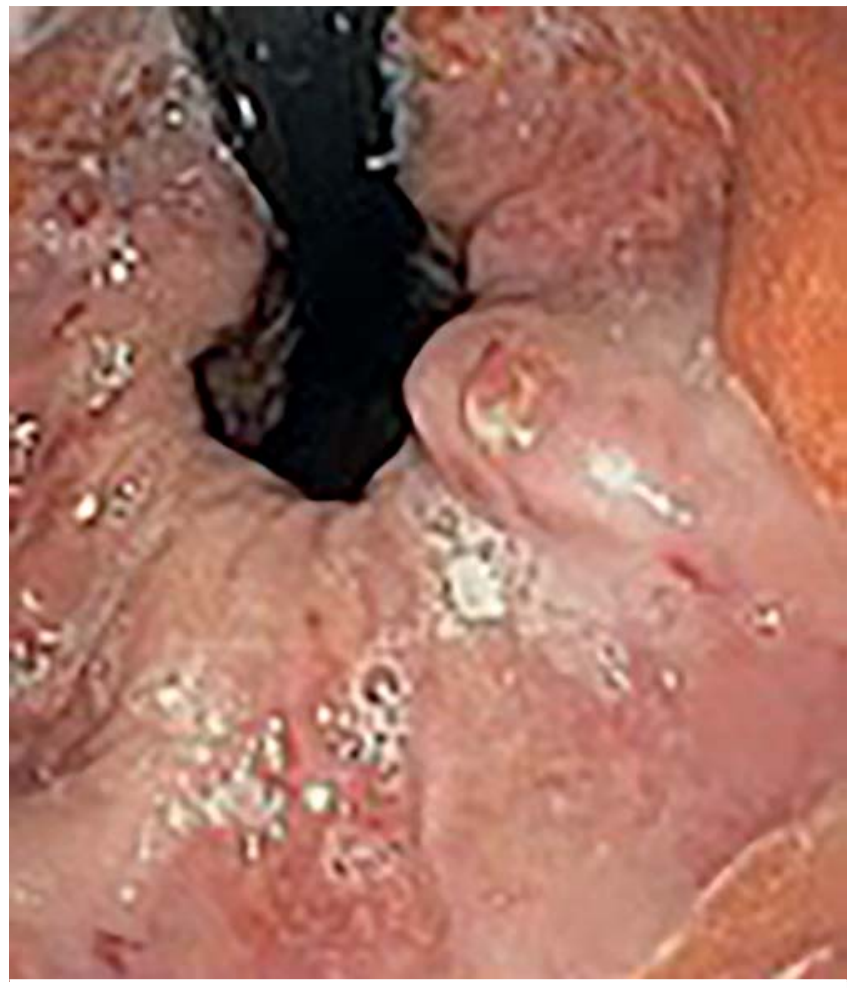

Fig. 3 Varices showing white nipple sign at different locations.

before June 2019 when elective intubation was not practiced. Three aspiration events still occurred when elective intubation was practiced as these patients had active spurt between the time when the scope was removed and when intubation was complete. Of the 631 patients in the nipple group, four died within 48 hours post-procedure $(0.5 \%)$ compared with four of $1970(0.2 \%)$ in the non-nipple group $(P=0.088)$.

- Table 1 Comparative data on positive nipple sign in 631 patients within the remaining cohort of 1970 patients.

\begin{tabular}{|l|l|l|}
\hline & Positive white nipple sign & Non-nipple group \\
\hline N & 631 & 1970 \\
\hline Active spurt during endoscopy & $137(21.7)$ & $210(10.2)$ \\
\hline Endotracheal Intubation needed & $78(12.3)$ & $112(5.7)$ \\
\hline Aspiration-related complications & $42(6.7)$ & $59(0.3)$ \\
\hline EVL successful & $594(94.1)$ & $1950(98.9)$ \\
\hline Glue in esophageal varices & $35(5.6)$ & $12(0.6)$ \\
\hline Mortality & $4(0.6)$ & $4(0.2)$ \\
\hline Placement of Denis Ella stent & $2(0.3)$ & 0 \\
\hline $\begin{array}{l}\text { Values presented as N (column \%). } \\
P \text { values are derived from chi square tests. Those }<0.05 \text { are considered to be significant. }\end{array}$ \\
\hline
\end{tabular}




\section{Discussion}

A positive white nipple sign is also known as a Mount St. Helen's sign. Dislodgement of this white nipple results in active spurt from a varix. This has been compared with the eruption of Mount St. Helen's. Although variceal bleeding is a common event, very limited literature exists regarding the white nipple sign. In our practice, we identified the sign in $24.3 \%$ of cases overall. Siringo et al. reported a white nipple in $9 \%$ of admissions for variceal bleeding [5]. Chung and Lewis found it in 5\% [2]. Thus, it is not a very uncommon feature, but mostly overlooked.

Siringo et al. found no difference in rebleeding in patients with and without a white nipple [5]. They concluded that the presence of a white nipple had no prognostic significance. We found no difference in mortality in the first 48 hours. However, these patients had a higher chance of onset of active spurt during endotherapy, thus making the procedure difficult. Risk of aspiration pneumonitis also increased in these patients. Withdrawal of the scope and elective intubation before proceeding for endotherapy can help decrease these complications, as was seen with the reduced occurrence of aspiration-related events after 2019 when we adopted this strategy.

By Injecting glue into the GOV1 and GOV2 varices coming up into the esophagus and feeding the varix with nipple and applying the first band on the varix with a white nipple sign, we successfully manage $94.1 \%$ our patients. Whether applying a band distal to the white nipple would have resulted in a different outcome is unknown.

Of our patients, $21.7 \%$ bled intraprocedurally. This happened possibly due to retching during sedation, friction between the scope and the nipple, or a very large varix with a thin wall. Once the bleed started from the nipple, endoscopic vision deteriorated and the situation became panicky. The chances of blood regurgitating and causing aspiration increased tremendously. Intubating all patients with a positive white nipple sign prior to proceeding to endotherapy prevents aspiration and allows deeper sedation, decreasing chances of a retch-induced spurt from the nipple.

We used glue for rescue when banding failed. Glue injection in the esophagus traditionally has been because of fear of complications such as fistula formation. Cipolletta et al. used glue in bleeding esophageal varices (52 with active bleed, 81 with stigmata of recent hemorrhage) and showed good efficacy without any major adverse events [6]. In our experience, we have found that these patients have mucosal bridging and fibrosis. We have not encountered fistula or sinus formation yet. We succeeded in all but two of our cases $(0.3 \%)$. As a last resort, we placed fully covered self-expanding covered metal stents (Denis Ella) to create tamponade and achieve hemostasis.

\section{Conclusions}

A positive white nipple sign on esophageal varices is not only a predictor of recent bleed, but also signifies high risk of intraprocedural bleed and it has to be managed with more caution and with a specific strategy.

\section{Competing interests}

The authors declare that they have no conflict of interest.

\section{References}

[1] Beppu K, Inokuchi K, Koyanagi N et al. Prediction of variceal hemorrhage by esophageal endoscopy. Gastrointest Endosc 1981; 27: 213218

[2] Chung RS, Lewis JW. The platelet-fibrin plug in esophageal variceal hemorrhage: the Mount St. Helens' sign. Gastrointest Endosc 1984; 30: 270

[3] Hou MC, Lin HC, Kuo BI et al. Clinical implications of the white nipple sign and its role in the diagnosis of esophageal variceal hemorrhage. Am J Gastroenterol 1996; 91: 2103-2109

[4] Desai P, Patel C, Kabrawala M et al. Use of n-butyl 2 cyanoacrylate without lipiodol, using a modified protocol in gastric variceal bleed management: retrospective analysis of 2299 patients. J Dig Endosc 2020; 11: 187-192

[5] Siringo S, McCormick PA, Mistry P et al. Prognostic significance of the white nipple sign in variceal bleeding. Gastrointest Endosc 1991; 37 : $51-55$

[6] Cipolletta L, Zambelli A, Bianco MA et al. Acrylate glue injection for acutely bleeding oesophageal varices: A prospective cohort study. Dig Liver Dis 2009; 41: 729-734 